\title{
Analysis of laser-generated plasma ionizing radiation by synthetic single crystal diamond detectors
}

\author{
M. Marinelli ${ }^{a}$, E. Milani ${ }^{a}$, G. Prestopino ${ }^{a}$, C. Verona ${ }^{\mathrm{a}, *}$, G. Verona-Rinati $^{\mathrm{a}}$, M. Cutroneo $^{\mathrm{b}}$, L. Torrisi $^{\mathrm{b}}$, \\ D. Margarone ${ }^{\mathrm{c}}$, A. Velyhan ${ }^{\mathrm{c}}, \mathrm{J}$. Krasa ${ }^{\mathrm{c}}$, E. Krousky ${ }^{\mathrm{c}}$ \\ a Dip. di Ing. Meccanica, Università di Roma “Tor Vergata," Roma 00133, Italy \\ ${ }^{\mathrm{b}}$ Dip. di Fisica, Università di Messina, S. Agata 98166, Italy \\ ' Institute of Physics of ASCR, Prague, Czech Republic
}

\section{A R T I C L E I N F O}

\section{Article history:}

Available online 15 June 2012

\section{Keywords:}

Single crystal diamond

Diamond detector

Laser-generated plasma

Ionizing radiation

Time-of-fight spectrometer

\begin{abstract}
A B S T R A C T
Diamond based detectors have been used in order to analyze the ionizing radiation emitted from the laser-generated plasma. High energy proton/ion beams were generated at Prague Asterix Laser System (PALS) Centre by the sub-nanosecond kJ-class laser at intensities above $10^{16} \mathrm{~W} / \mathrm{cm}^{2}$.

The tested detectors consisted of a photoconductive device based on high quality chemical vapor deposition (CVD) single crystal diamond, produced at Rome "Tor Vergata" University. They have been operated in planar configuration, having inter-digitized electrodes.

The proposed diamond detectors were able to measure UV, X-rays, electrons and ions. They have been employed in time-of-flight (TOF) configuration and their reliability was checked by comparison with standard ion collectors (mostly used at PALS). Both the forward and backward expanding plasma was characterized in the experiment. The results indicate that diamond detectors are very promising for the characterization of fast proton and ion beams produced by high power laser systems.
\end{abstract}

() 2012 Elsevier B.V. All rights reserved.

\section{Introduction}

The investigation of fast ion emission from laser plasmas is crucial for future applications of laser-accelerated ion beams in different areas, such as warm dense matter generation, probing of high electric and magnetic fields, nuclear applications (compact neutron sources, isotope production, fission reactions), medicine, astrophysics, etc. Recently, the generation of ions with energy of few $\mathrm{MeV} / \mathrm{u}$ and current densities of few $\mathrm{A} / \mathrm{cm}^{2}$ by the use of the subnanosecond, kJ-class laser at Prague Asterix Laser System (PALS) laboratory has been reported [1-3]. The beam parameters are estimated with application of various devices and techniques usually operated in "single shot" mode. In particular, time-of-flight (TOF) spectrometry is an effective diagnostic tool [4] since it provides time-resolved measurements which are fundamental for a detailed study of ion beam parameters, such as species and charge-states, their kinetic energy, and total charge. However, there exist various difficulties in such diagnostic techniques when high-energy laser pulses are used, e.g. high electromagnetic noise, overlapping of the signal due to photopeak, difficulties in separation of the ion signals from it, breakdowns at very high ion currents, low sensitivity to fast electrons and protons or to low charge state ions. To overcome

\footnotetext{
* Corresponding author.

E-mail address: claudio.verona@uniroma2.it (C. Verona).
}

the above measurement difficulties, the development of new detectors is in continuous growing. In particular, semiconductor detectors seem to be very promising for detection of fast protons and ion beams produced by high power laser systems [5-7]. Among them, the use of diamond detectors, producing signals proportional to the energy deposited by the incident radiation, is very attractive because these detectors are not sensitive to the visible light (band gap $5.48 \mathrm{eV}$ ). Moreover, high thermal conductivity, high resistivity, low dielectric constant, high carrier mobility and radiation hardness $[8,9]$ suggest that diamond is an ideal material for monitoring the radiation emission from laser-generated plasmas. Thanks to the fast response time, detectors based on natural and synthetic diamond have been employed in TOF techniques in several experiments reported in literature, in particular for the detection of electrons [10] and heavy ions [11]. Detectors based on high quality chemical vapor deposition (CVD) single crystal diamond have been developed at the Department of Mechanical Engineering of University of Rome "Tor Vergata" for both nuclear particles (thermal and fast neutrons, high energy ions, charged particles) [12] and photon radiations (X-rays, UV, etc.) [13]. Such diamond detectors have been permanently installed at Joint European Torus (JET) to measure $\mathrm{UV} / \mathrm{X}$-ray radiation and neutrons produced by JET plasma demonstrating a fast time response, good sensitivity, high signal-to-noise ratio and a good stability and reliability of the response [14].

In this work, single-crystal CVD diamond detectors are employed in TOF technique to monitor the different plasma 
products (i.e. photons, electron and ions) generated by high power pulses focalized on different targets at PALS laboratory in Prague. Both forward and backward emission of the plasma was characterized. In particular, different thin hydrogenated targets were used in the experiment in order to generate forward protons, i.e., proton emission from the rear target sides. A different electrode configuration was adopted for the single crystal diamond detectors with respect to Refs. [12-14] in order to improve the time resolution and better separate the different reaction products.

In this work, single-crystal CVD diamond detectors operating in planar configuration are employed in TOF technique to monitor the emission of photons, electron and ions ejected from both forward and backward emission of the plasma produced at PALS laboratory in Prague.

\section{Material and methods}

The diamond detectors were fabricated in a planar interdigitized electrode configuration. A nominally intrinsic diamond layer, which operates as the detecting region, was homoepitaxially grown by Microwave Plasma Enhanced Chemical Vapor Deposition (MWPECVD) on a $4 \mathrm{~mm} \times 4 \mathrm{~mm} \times 0.5 \mathrm{~mm}$ commercial low-cost synthetic High Pressure High Temperature (HPHT) diamond substrate. The thickness of the intrinsic layer was chosen to be approximately $50 \mu \mathrm{m}$. The hydrogen termination of the as grown diamond surface was removed by isothermal annealing at $500^{\circ} \mathrm{C}$ for $1 \mathrm{~h}$ in air. Finally, $50 \mathrm{~nm}$ thick aluminum fingers were patterned by a standard lift-off photo-lithographic technique and by thermal evaporation. The metal fingers were processed to $5 \mu \mathrm{m}$ in width with spacing between the electrodes of $20 \mu \mathrm{m}$. The detector active area was approximately $8 \mathrm{~mm}^{2}$. Such a structure, operating in planar configuration, acts as a two terminal metal-diamond-metal (MDM) device. An external voltage of $50 \mathrm{~V}$ was applied between the metal contacts generating an electric field perpendicular to the incoming light across the device. Mobile charges produced by the absorbed radiation drift in this electric field and generate a current in the external circuit. From simulations based on the Shockley-Ramo-Gunn theory with the help of Comsol Multiphysics finite element software [15], the charge collection efficiency of the MDM detector is such to allow collecting electron-hole pairs generated up to about $25 \mu \mathrm{m}$ below the superficial metallic electrodes. So that the diamond substrate, which is poor in electrical quality, does not contribute to the detection process. The sensitive layer is thick enough to collect most of the energy of the emitted ions and, in particular, protons of energy up to $2 \mathrm{MeV}$ are fully captured in this layer (SRIM Monte Carlo code [16]).

In order to reduce electromagnetic noise, the diamond detectors were placed in a shielded holder having a pin-hole to collimate the radiation only on the detector sensitive area. The output signal was recorded with a $1 \mathrm{GHz}, 5 \mathrm{GS} / \mathrm{s}$ fast oscilloscope through a biasT. All inputs to scope were terminated in $50 \Omega$. Fig. 1 shows the schematic structure of the MDM device with the scheme of the used electronic circuit (a), a photo of $\mathrm{Al}$ inter-digitized electrodes (b) and its assembly in the experiment (c). The diamond detectors were employed in TOF measurements to detect all the components of the radiation emitted from the laser-generated plasma.

The experiment has been performed at the PALS facility by focusing the first harmonics $\left(\lambda_{0}=1315 \mathrm{~nm}\right)$ of the $300 \mathrm{ps}$ pulsed laser at a maximum laser energy of $600 \mathrm{~J}$ and laser intensity above $10^{16} \mathrm{~W} / \mathrm{cm}^{2}$. Thick and thin metal layers or polymers were employed as targets. Moreover, multilayered films, most of them constituted by hydrogenated polymers coupled to thin metallic films (Mylar/Al, Al/Mylar/Al, Au/polyethylene, polymers containing nanoparticles and/or nanostructures, etc.), were used in order to increase the energy of ejected ions. Investigation of proton and

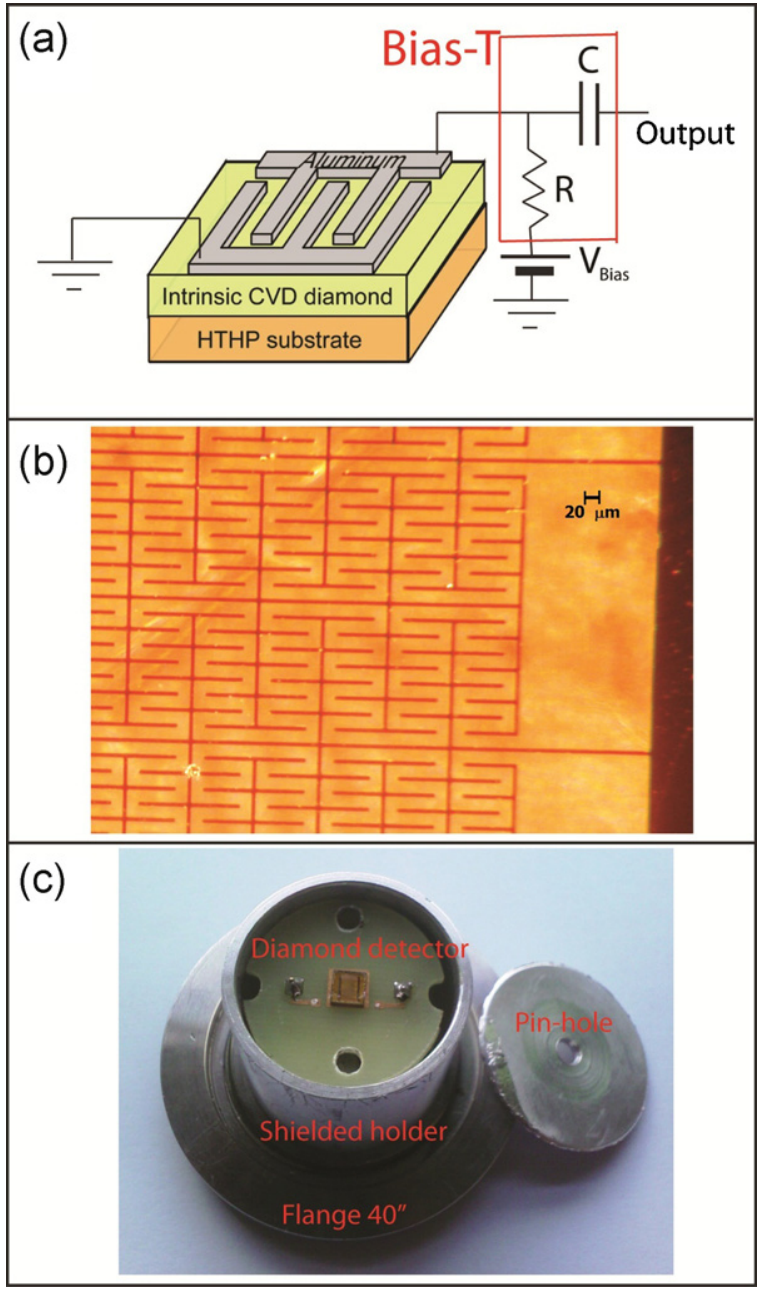

Fig. 1. Schematic structure of the MDM device with the scheme of the used electronic circuit $(\mathrm{R}=3.3 \mathrm{M} \Omega$ and $\mathrm{C}=0.68 \mu \mathrm{F})(\mathrm{a})$, a photo of $\mathrm{Al}$ inter-digitized electrodes (b) and its assembly in the experiment (c).

ion emissions in backward and forward directions (thick and thinner targets, respectively) of the plasmas was analyzed. Forward emission of ions (mainly protons) was measured from the rearside (target back side) of thin targets along the normal to the target surface, besides backward emission of the plasma is from the front side of the target.

The MDM detectors were placed at a distance of $101 \mathrm{~cm}$ and $123 \mathrm{~cm}$ from the target surface in forward and backward directions, respectively, and at $30^{\circ}$ angle with respect to the target normal direction in both cases. A simple sketch of the experimental setup is shown in Fig. 2.

Finally, a Faraday Cup was employed as an ion collector (IC) at a distance of $100 \mathrm{~cm}$ from the target in forward direction and at $30^{\circ}$ angle in order to compare the experimental data obtained from Diamond with that from IC.

\section{Results and discussion}

First, the time resolution analysis of the MDM detectors has been performed in our laboratories. Then, the results obtained by two photodetectors, one placed in forward and another one in backward direction of the plasma at PALS laboratory, are reported in the following. 


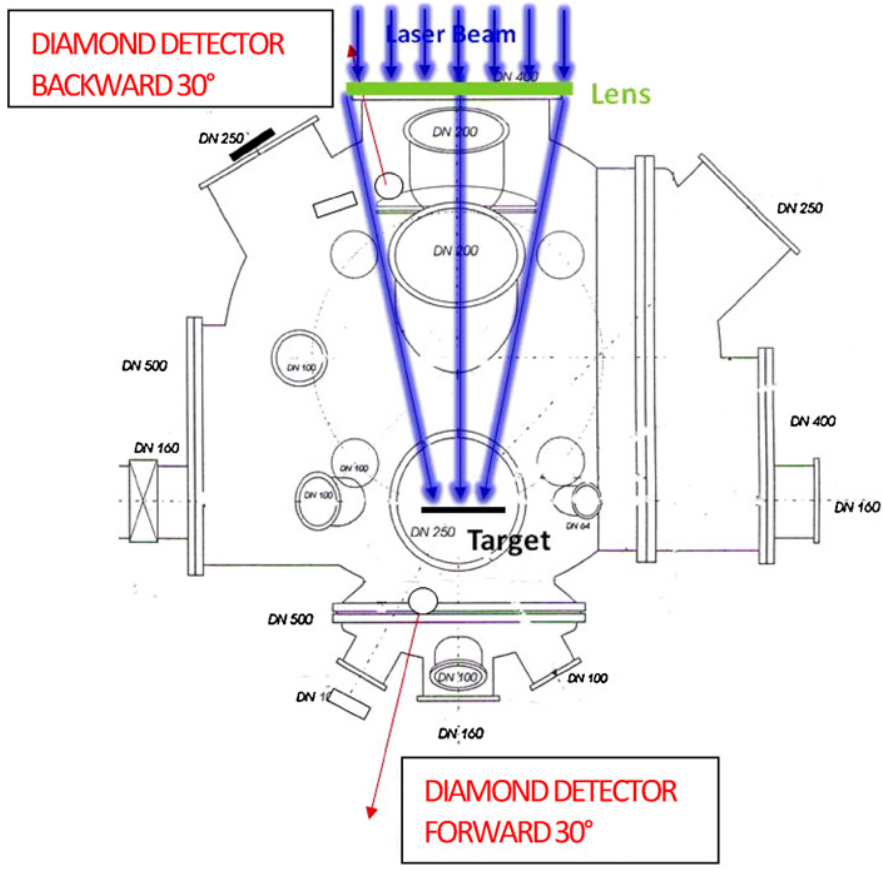

Fig. 2. Photo and scheme of the experimental irradiation vacuum chamber at PALS laboratory.

\subsection{Time response}

The high saturation velocity and high mobility of charge carriers in diamond makes the MDM detector's response very fast. In the case of homogeneous electric field $E_{\mathrm{d}}$ condition and neglecting any recombination and capture (trapping), the effective charge collection time $(\tau)$ of electron-hole pairs generated in the diamond across the electrodes is given by [17]

$$
\begin{aligned}
& \tau=\frac{\mu_{e} \cdot \tau_{e}+\mu_{h} \cdot \tau_{h}}{\mu_{e}+\mu_{h}} \\
& \tau_{e / h}=\frac{d}{\mu_{e / h} E_{d}} \cdot\left(1+\frac{\mu_{e / h} E_{d}}{v_{s a t}(e, h)}\right)
\end{aligned}
$$

where $d$ is the distance between the electrodes, $\mu_{e / h}$ is the charge carrier mobility and $v_{\text {sat }(e, h)}$ is the saturation velocity. Using the values $d=20 \mu \mathrm{m}, \mu_{e}=1714 \mathrm{~cm}^{2} \mathrm{~V}^{-1} \mathrm{~s}^{-1}, \mu_{h}=2064 \mathrm{~cm}^{2} \mathrm{~V}^{-1} \mathrm{~s}^{-1}$, $E_{d}=2 \times 10^{4} \mathrm{~V} \mathrm{~m}^{-1}$ (drift field) and $v_{\text {sat }(e)}=9.6 \times 10^{6} \mathrm{~cm} \mathrm{~s}^{-1}$, $v_{\text {sat }(h)}=1.4 \times 10^{7} \mathrm{~cm} \mathrm{~s}^{-1}[18,19]$, Eq. (1) gives $\tau \approx 200 \mathrm{ps}$. In order to study the effective time response of the MDM detectors, the devices are have been irradiated with $5.5 \mathrm{MeV}$ alpha particles (13 $\mu \mathrm{m}$ penetration depth in diamond [16]). The detector was placed at approximately $1 \mathrm{~cm}$ in front of the ${ }^{241} \mathrm{Am}$ source. For such measurement, a low noise Diamond Broadband Amplifier (DBA-III) [20] was used as fast front-end electronics coupled to a digital fast scope ( $2.3 \mathrm{GHz}$ bandwidth and $50 \mathrm{GHz}$ sampling rate). Charged $\alpha$ particles passing through the diamond detector produce short electrical pulses. A pulse from $5.5 \mathrm{MeV}$ alpha particles measured by diamond detector is shown in Fig. 3 varying the bias applied to the detector. From the Gaussian fit of the pulses, about 350 ps full width at half maximum (FWHM) is observed when the applied bias is higher than $40 \mathrm{~V}$ demonstrating a high intrinsic response velocity of the MDM detectors. However, the discrepancy between the theoretical value calculated above and the experimental one is probably to be ascribed to the approximations, e.g. the non uniformity of the electric field in the bulk of MDM detector, trapping, etc.

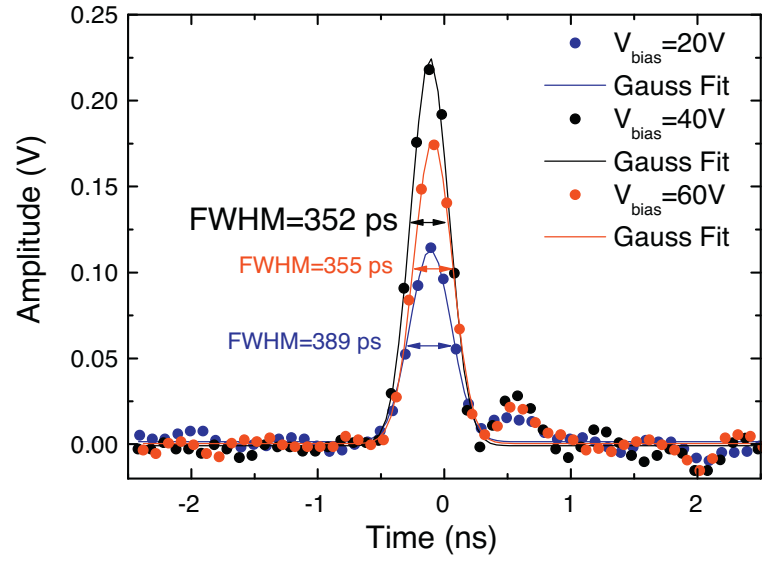

Fig. 3. A pulse from $5.5 \mathrm{MeV}$ alpha particles obtained with the DBA-III preamplifier and recorded with a digital fast oscilloscope.

\subsection{Time-of-flight measurements at Pals}

A typical TOF spectrum measured by the diamond detector in backward emission of the plasma is shown in Fig. 4. This spectrum is obtained by irradiating the polyethylene terephthalate (PET) target material at $1064 \mathrm{~nm}, 494 \mathrm{~mJ}$ pulse energy and with the laser focus exactly on the target surface. The MDM detector is able to discriminate different components of the radiation emitted from the target. In particular, the following peaks can be seen vs. time: at the beginning of the scale, the photo-peak arising from UV and $\mathrm{X}$-ray radiation (0-20 ns), the peaks arising from fast plasma electrons (20-100 ns), the peak arising from fast protons (120 ns), the peaks from different ion species and ionization stages (190-400 ns) and the peaks arising from slow ions $(0.4-1 \mu \mathrm{s})$ emitted from the hot target material surrounding the plasma spot. These different plasma products (radiation and particles) were assigned to each peak according to previous results $[7,21]$ and also thanks to simultaneous measurements by particle spectrometers employed in the experiment but not reported in this work. The conversion of TOF into the kinetic energy $E_{p}$ is determined by the following equation using the relativistic kinematics

$$
\begin{aligned}
& E_{p}=\frac{1}{2} M_{0} c^{2} \cdot\left(\frac{1}{\sqrt{1-\beta^{2}}}-1\right) \\
& \beta=\frac{d}{c \cdot T O F}
\end{aligned}
$$

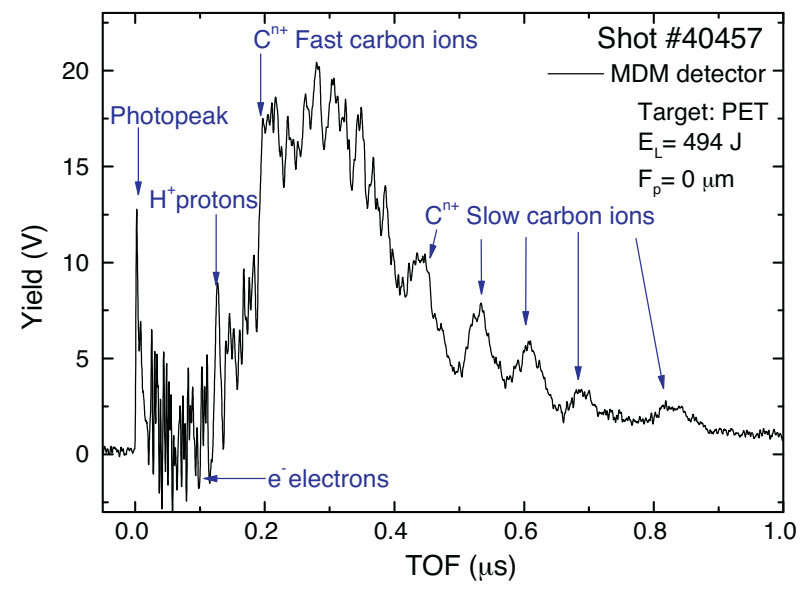

Fig. 4. Typical TOF spectra measured by diamond detector in backward emission of the plasma. 


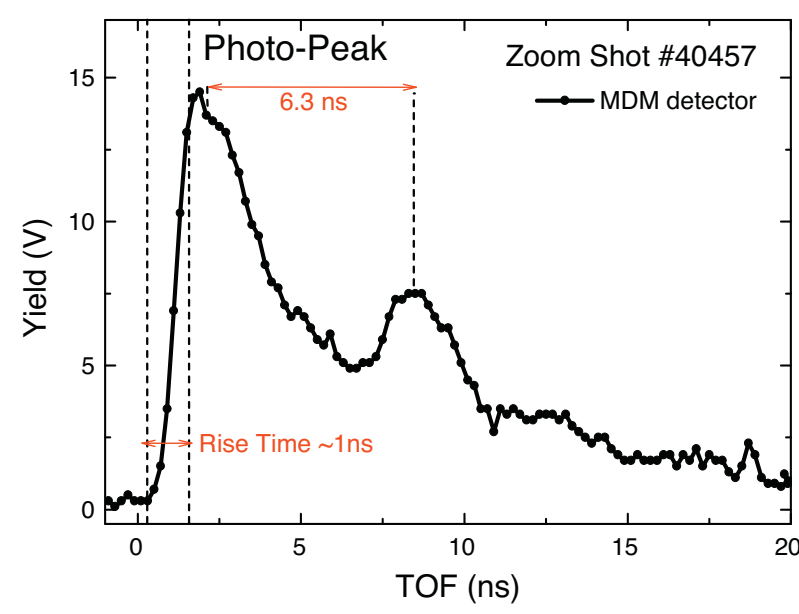

Fig. 5. Enlarged view of the photo-peak of the spectrum reported in Fig. 4.

where $M_{0}$ is the rest mass of a proton, electron or ion, $c$ is the light velocity and $d$ is the flight path (i.e. the target-diamond detector distance). The spectrum indicates a proton energy of about $280 \mathrm{keV}$ and a carbon ion energy ranging between about $295 \mathrm{keV}$ and $11 \mathrm{MeV}$. In the portion of the spectrum between 50 and $100 \mathrm{~ns}$, the photodetector response is not affected by noise but an additional and opposite polarity signal due to contribution of secondary electron emission arising from the metallic interdigitated contacts interferes when low energetic electrons are incoming to the diamond surface. Such electrons, have an energy of the order of $2.8 \mathrm{keV}-500 \mathrm{eV}$, confirming that the hotter generated plasma may reach a temperature of the order of $2 \mathrm{keV}$, in agreement with literature [21]. An enlarged view of the TOF spectrum discussed above is reported in Fig. 5. Because the diamond is not sensitive to the plasma visible and soft-UV radiation up to $225 \mathrm{~nm}$, a short photopeak with a temporal decay of about $15 \mathrm{~ns}$ and a rise time of $1 \mathrm{~ns}$ can be observed. A second peak about 6 ns after is well visible, demonstrating the high time resolution of the detector. Such a peak can possibly be ascribed to a fast plasma electron component with an average energy of about $50 \mathrm{keV}$.

Two typical TOF spectra obtained at PALS in the forward direction (about 500 J laser energy, thin Mylar and $\mathrm{Al} / \mathrm{Mylar} / \mathrm{Al}$ ) are reported in Fig. 6(a) and (b). The TOF diamond spectra were compared with traditional ion collector spectra. The $x$-axis was normalized at a distance of $100 \mathrm{~cm}$ in order to perform a direct comparison of the TOF spectra simultaneously recorded with the two different detectors. In all cases, the IC spectra show a huge photopeak with a tail lasting about $0.5 \mu$ s so that the signal related to fast ions and protons is completely hidden by such peak. However, it shows a high efficiency for the low-energy ions between 1 and $4 \mu \mathrm{s}$ of the TOF spectra. These slow $\mathrm{C}$ and $\mathrm{Al}$ ions at different charge state and energy are also measured by the diamond detector as clearly seen in Fig. 6(a) and in the inset of Fig. 6(b). In fact, the slow ions do not lose energy in the front electrode since the MDM detectors, operating in a planar configuration, have its active diamond volume directly exposed to impinging radiation. The $\mathrm{Cn}^{+}$mean energy measured by the ICR $(30 \mathrm{keV})$ is slightly lower than the one measured by the diamond detector ( $40 \mathrm{keV}$ ), since its annular geometry does not allow to measure the faster ion stream passing on axis where the diamond detector is placed. In fact, in a laser plasma the higher energy ion streams are typically more collimated (smaller aperture cone) $[22,23]$. Moreover, the proton peak is well separated from the photo-peak, which is quite narrow, and give the possibility of measuring very well fast protons and ions. Finally, MDM detector reveals also electrons, whose peaks are between the photo-peak and proton peak. It is possible to conclude that by using the
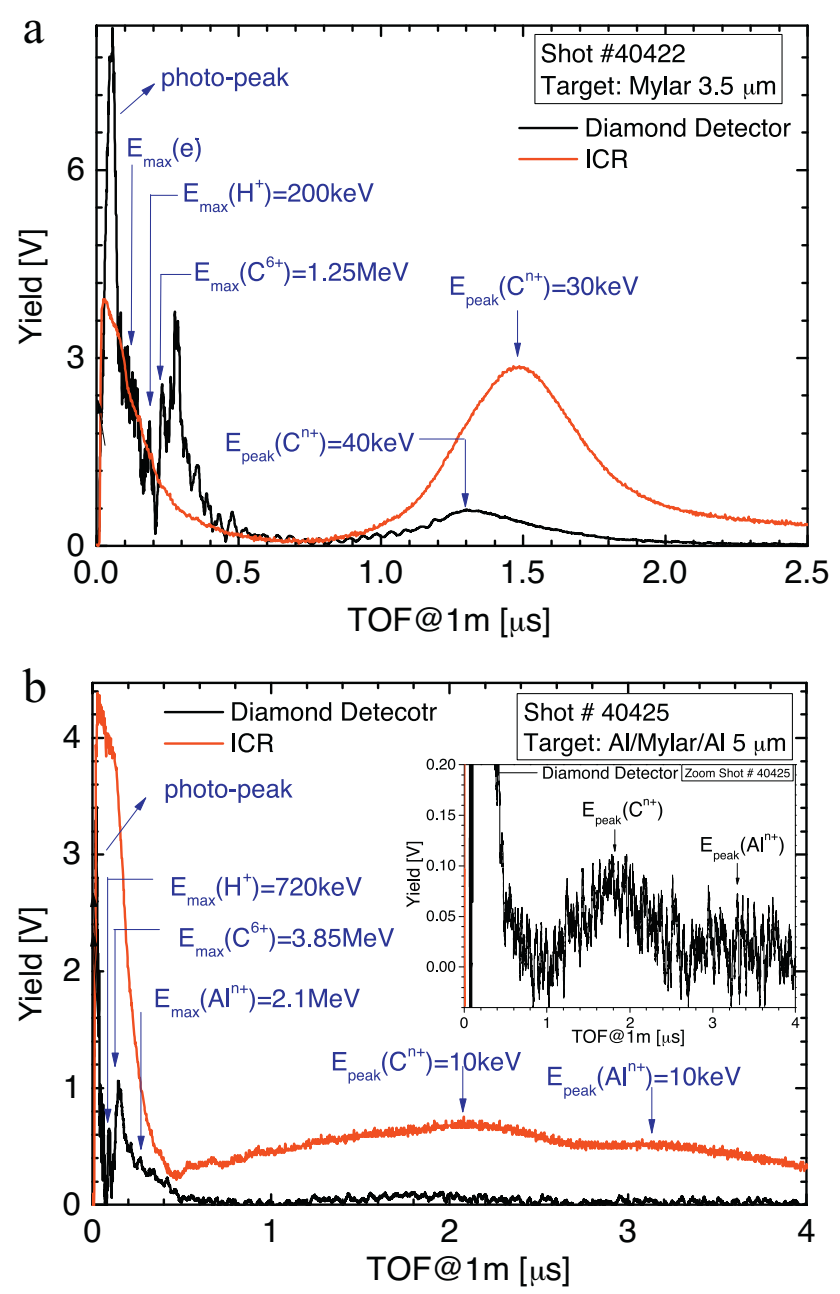

Fig. 6. Typical diamond TOF spectra for diamond and ion collector obtained by ablating different thin targets at PALS laboratory in forward direction.

diamond detectors in TOF configuration, it is easier to emphasize the fast ion peak and electrons at expenses of the relatively short XUV photopeak respect to the IC detector which is instead more efficient to measure slow ions, since it has a lower sensitivity to the high energy, low charge stat particles, and also because it is very sensitive to the soft-UV plasma radiation which overlaps with the fast ion peak [24].

\section{Conclusion}

Single-crystal CVD diamond detectors with an interdigitated metal electrode have been fabricated and tested at "Tor Vergata" Rome University. Such diamond detectors have been employed in time-of-flight (TOF) configuration to monitor the emission plasma obtained at PALS laboratory in Prague by using a laser pulse intensity of about $10^{16} \mathrm{~W} / \mathrm{cm}^{2}$.

The achieved results demonstrated that diamond detectors show important advantages with respect to other semiconductor detectors. These include the absence of a response to visible light and the possibility to simultaneously detect energetic photons, electrons, and ions ejected from the plasma both in forward and backward directions. Finally, diamond detectors have a good sensitivity to fast ions and high energy resolution (fast response time) which is a good advantage when it is necessary to distinguish between the accelerated fast protons and the relatively long plasma photopeak. 


\section{Acknowledgements}

This work is supported by LIANA-INFN Experiment and partially by the Fondazione Roma.

\section{References}

[1] L. Laska, J. Kársa, A. Velyhan, K. Jungwirth, E. Krouský, D. Margarone, M. Pfeifer, K. Rohlena, L. Ryc, J. Skála, L. Torrisi, J. Ullschmied, Laser and Particle Beams 27 (2009) 137.

[2] J. Krasa, A. Velyhan, D. Margarone, E. Krouský, J. Ullschmied, J. Skala, L. Laska, K. Jungwirth, K. Rohlena, Review of Scientific Instruments 81 (2010) 02 A504.

[3] D. Margarone, J. Krasa, L. Laska, A. Velyhan, T. Mocek, J. Prokupek, E. Krousky, M. Pfeifer, S. Gammino, L. Torrisi, J. Ullschmied, B. Rus, Review of Scientific Instruments 81 (2010) 02A506.

[4] E. Woryna, P. Parys, J. Wolowski, M. Mroz, Laser and Particle Beams 14 (1996) 293.

[5] M. Farnikova, J. Krasa, B. Kralikova, J. Skala, P. Parys, L. Ryc, J. Wolowski, E. Woryna, Proceedings of the SPIE: The International Society for Optical Engineering 2767 (1996) 113.

[6] L. Torrisi, G. Foti, L. Giuffrida, D. Puglisi, J. Wolowski, J. Badziak, P. Parys, M. Rosinski, D. Margarone, J. Krasa, A. Velyhan, U. Ullschmied, Journal of Applied Physics 105 (2009) 123304.

[7] L. Torrisi, D. Margarone, L. Laska, M. Marinelli, E. Milani, G. Verona Rinati, S. Cavallaro, L. Ryc, J. Krasa, K. Rohlena, J. Ullschmied, Journal of Applied Physics 103 (2008) 083106.

[8] J.E. Field, Properties of Diamond, Academic, London, 1979.

[9] D.R. Kania, M.I. Landstrass, M.A. Plano, L.S. Pan, S. Han, Diamond and Related Materials 2 (1993) 1012.

[10] A.V. Krasilnikov, K.N. Makarov, I.N. Rastyagaev, Yu.A. Satov, Yu.B. Smakovskiy, A.G. Alekseyev, V.N. Amosov, Review of Scientific Instruments 72 (2001) 1258.
[11] E. Berdermann, K. Blasche, P. Moritz, H. Stelzer, B. Voss, Diamond and Related Materials 10 (2001) 1770.

[12] S. Almaviva, M. Marinelli, E. Milani, G. Prestopino, A. Tucciarone, C. Verona G. Verona-Rinati, M. Angelone, D. Lattanzi, M. Pillon, R.M. Montereali, M.A Vincenti, Journal of Applied Physics 103 (2008) 054501

[13] S. Almaviva, M. Marinelli, E. Milani, G. Prestopino, A. Tucciarone, C. Verona, G. Verona-Rinati, M. Angelone, M. Pillon, I. Dolbnya, K. Sawhney, N. Tartoni, Journal of Applied Physics 107 (1) (2010) 014511.

[14] M. Angelone, M. Pillon, M. Marinelli, E. Milani, G. Prestopino, C. Verona, G. Verona-Rinati, I. Coffey, A. Murari, N. Tartoni, Nuclear Instruments and Methods in Physics Research Section A 623 (2010) 726.

[15] Consol MultiPhysics website: http://www.comsol.com/.

[16] J.F. Ziegler, J.P. Biersack, M.D. Ziegler, SRIM-The Stopping and Range of Ions in Matter, SRIM, Annapolis, MD, 2008.

[17] G.F. Knoll, Radiation Detection and Measurement, 3rd ed., Wiley, New York 2000.

[18] M. Pomorski, E. Berdermann, M. Ciobanu, A. Marte-myianov, P. Moritz, M. Rebisz, B. Marczewska, Physica Status Solidi A 202 (2005) 11.

[19] H. Pernegger, S. Roe, P. Weilhammer, V. Eremin, H. Frais-Kolbl, E. Griesmayer, H Kagan, S. Schnetzer, R. Stone, W. Trischuk, D. Twitchen, A. Whitehead, Journal of Applied Physics 97 (2005) 173704

[20] P. Moritz, E. Berdermann, K. Blasche, H. Stelzer, B. Voss, Diamond and Related Materials 10 (2001) 1765.

[21] D. Margarone, J. Krasa, L. Giuffrida, A. Picciotto, L. Torrisi, T. Nowak, P. Musumeci, A. Velyhan, J. Prokpek, L. Lska, T. Mocek, J. Ullschmied, B. Rus, Journal of Applied Physics 109 (2011) 103302.

[22] L. Torrisi, D. Margarone, Plasma Sources Science and Technology 15 (2006) 635

[23] T.E. Cowan, J. Fuchs, H. Ruhl, A. Kemp, P. Audebert, M. Roth, R. Stephens, Barton, A. Blazevic, E. Brambrink, et al., Physical Review Letters 92 (2004) 204801.

[24] L. Torrisi, S. Gammino, L. Andò, L. Laska, J. Krasa, K. Rohlena, J. Ullschmied, J. Wolowski, J. Badziak, P. Parys, Journal of Applied Physics 99 (2006) 083301. 